\title{
Combining thioridazine and loratadine for the treatment of gastrointestinal tumor
}

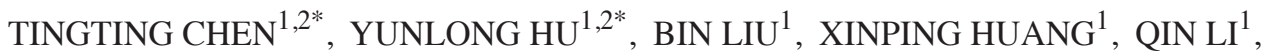 \\ NINGNING GAO ${ }^{1}$, ZHENCHAO JIN ${ }^{1}$, TIELIU JIA ${ }^{1,2}$, DEYIN GUO ${ }^{2}$ and GUANGYI JIN ${ }^{1}$ \\ ${ }^{1}$ Cancer Research Center, Department of Medicine, Shenzhen University, Shenzhen, Guangdong 518060; \\ ${ }^{2}$ Department of Basic Medical Sciences, Wuhan University, Wuhan, Hubei 430071, P.R. China
}

Received January 4, 2016; Accepted June 15, 2017

DOI: $10.3892 / \mathrm{ol} .2017 .6815$

\begin{abstract}
In 2015, the American Society of Clinical Oncology announced that strategies of using combination therapies have been indicated to be effective against many types of cancer. In the present study, thioridazine (THZ) was used in a combination therapy with loratadine (LOR) to target gastrointestinal tumor, with the aim of investigating whether combined therapy was superior to monotherapy in its antitumor effects. The antiproliferative effects on CT26.WT and MFC cells were analyzed using cell-counting kit-8 assay, and synergistic effect was assessed by combination index (Fig. 1). Annexin V and propidium iodide staining indicated the combination therapy was able to induce apoptosis and that this may be mediated via caspase-3, -9 and poly (ADP-ribose) polymerase (PARP) (Fig. 2). Antitumor activity was also evaluated in CT26.WT xenografts in BALB/c mice (Fig. 3). Furthermore, as expected, combination therapy was able to successfully inhibit the phosphoinositide 3-kinase/Akt/mammalian target of rapamycin signaling pathway (Fig. 4). These findings suggest that the combination therapy with THZ and LOR may provide a promising therapy for gastrointestinal cancer.
\end{abstract}

\section{Introduction}

In China, at present, $>60 \%$ of all malignant tumors are diagnosed to be in the digestive system, with colon and gastric cancer being the most frequently diagnosed types of

Correspondence to: Professor Guangyi Jin, Cancer Research Center, Department of Medicine, Shenzhen University, 3688 Nanhai Street, Shenzhen, Guangdong 518060, P.R. China

E-mail: gyjin@szu.edu.cn

Professor Deyin Guo, Department of Basic Medical Sciences, Wuhan University, 16 Luojiashan Road, Wuhan, Hubei 430071, P.R. China

E-mail:dguo@whu.edu.cn

*Contributed equally

Key words: thioridazine, loratadine, antipsychotic drug, gastrointestinal tumor, apoptosis cancer (1). In 2008, colon cancer was the third most common cancer in males and the second in females with $>1.2$ million novel cases diagnosed (2). Additionally, gastric cancer was the fourth most common cancer worldwide, with a total of 989,600 novel cases of stomach cancer in 2008 (2). Although the rates of mortality in gastric cancer have declined in a number of developed countries, mainly due to improvements in quality of life and treatment, high mortality rate and poor prognosis remain on the increase in many developing countries (2). Conventional therapies involving surgery, chemotherapy, and radiation therapy are often unable to eradicate all tumor cells and hence have low rates of efficacy (3). In 2015, the American Society of Clinical Oncology announced that strategies of using combination therapies have been indicated to be effective against many types of cancer (4). Successful examples of synergistic combination therapy may provide a novel approach for gastrointestinal cancer therapy (4).

There is a link between the nervous and digestive system (5). The digestive system is the most complicated nervous system other than the central nervous system, with hundreds of millions of independent nerve cells (6). Some drugs used in the treatment of neurological disorders are also often used to relieve gastrointestinal discomfort (7). Therefore, using drugs that target the nervous system to treat the digestive system cancer may provide novel treatment avenues.

Thioridazine (THZ), a phenothiazine derivative that acts as an antipsychotic drug, has been previously reported to selectively inhibit leukemic cancer stem cells (8). It was also reported that THZ is able to exhibit potent antitumor effects in gastric, breast, ovarian, cervical and endometrial cancer (9-12). THZ is also able to inhibit angiogenesis by targeting the vascular endothelial growth factor receptor 2/phosphoinositide 3-kinase (PI3K)/mammalian target of rapamycin (mTOR) signaling pathway in ovarian cancer xenografts (11). THZ has also been demonstrated to exhibit anti-cancer effects via the $\mathrm{PI} 3 \mathrm{~K} / \mathrm{Akt} / \mathrm{mTOR}$ signaling pathway in cervical and endometrial cancer cells (12).

Loratadine (LOR), a well-known, oral anti-histamine used worldwide, has been reported to inhibit the growth of human colon cancer cells by inducing G2/M phase cell cycle arrest and apoptosis via caspase-9 (13).

Here, the authors of the present study hypothesized that THZ is able to mediate a synergistic effect with LOR for 
the treatment of gastrointestinal cancer, and the underlying molecular mechanisms were subsequently investigated.

\section{Materials and methods}

Cell culture, reagents and antibodies. Mouse colon cancer cells (CT26.WT) and mouse gastric cancer cells (MFC) were purchased from the Cell Bank of the Chinese Academy of Sciences (Shanghai, China), and maintained in RPMI-1640 medium (Thermo Fisher Scientific, Inc., Waltham, MA, USA) with $10 \%$ fetal bovine serum (FBS; Invitrogen; Thermo Fisher Scientific, Inc.), $100 \mathrm{U} / \mathrm{ml}$ penicillin/streptomycin (Invitrogen; Thermo Fisher Scientific, Inc.) in a humidified incubator with $5 \% \mathrm{CO}_{2}$ atmosphere at $37^{\circ} \mathrm{C}$. THZ and LOR were purchased from Sigma-Aldrich (Merck KGaA, Darmstadt, Germany). The following antibodies were used in the present study: Anti-Akt (cat. no. 9272), anti-phospho-specific Akt (cat. no. 4060), anti-PI3K (cat. no. 4249), anti-phospho-specific PI3K (cat. no. 4228), anti-mTOR (cat. no. 2983), anti-phospho-specific mTOR (cat. no. 5536), anti- $\beta$-actin (cat. no. 3700) and Cleaved Caspase Antibody Sampler kit (cat. no. 9929; all Cell Signaling Technology Inc., Danvers, MA, USA).

Cell-counting kit-8 (CCK-8). Inhibition of proliferation was quantified using CCK-8 (CK04; Dojindo Molecular Technologies, Inc., Kumamoto, Japan) following the manufacturer's instructions. A total of $1 \times 10^{4}$ cells were seeded in each well of 96-well plates overnight, and treated with THZ or $\operatorname{LOR}(1,5,10,25,50$ or $75 \mu \mathrm{M})$, or a combination of THZ and LOR. After $24 \mathrm{~h}, 10 \mu \mathrm{l}$ CCK-8 kit solution was added to each well. Subsequently, the cells were incubated for another $2 \mathrm{~h}$, and the absorbance at $450 \mathrm{~nm}$ was measured by using a microplate reader. Each individual measurement was repeated three times.

Evaluation for combination index (CI). The synergistic inhibitory effects of THZ and LOR were calculated according to the Chou-Talalay method (14). CI is a quantitative measure of the degree of interaction between drugs according to the absorbance of each group and is calculated as follows: $\mathrm{CI}=\mathrm{AB} /(\mathrm{A} \times \mathrm{B})$, where $\mathrm{AB}$ is the ratio of the combination groups to the control group and $\mathrm{A}$ or $\mathrm{B}$ is the ratio of the single group to the control group. A value of $\mathrm{CI}<1$ indicates synergism, and $\mathrm{CI}=1$ denotes additive effects. A value of $\mathrm{CI}>1$ indicates antagonism.

Apoptosis assay. A total of $5 \times 10^{5}$ cells were seeded in 6-well plates overnight. The cells were treated with $10 \mu \mathrm{M}$ THZ or $25 \mu \mathrm{M}$ LOR, or a combination of $10 \mu \mathrm{M}$ THZ and $25 \mu \mathrm{M}$ LOR respectively for $24 \mathrm{~h}$. The cells were then washed twice with PBS, and stained with Annexin V and propidium iodide (PI) in binding buffer for $15 \mathrm{~min}$, according to the manufacturer's protocols (BD Biosciences, Franklin Lakes, NJ,USA). The cells were then analyzed using flow cytometry (BD Biosciences).

Animals and mouse xenografts. A total of 30 female BALB/c mice (four- to six-weeks-old) were supplied by the Guangdong Medical Laboratory Animal Center (Guangzhou, China). The mice were housed in a temperature-controlled room with alternating $12 \mathrm{~h}$ light/dark cycles at $18-22^{\circ} \mathrm{C}$ and $40-70 \%$ humidity and fed a standard diet until euthanasia at the predetermined end-point, 35 days, when the tumors reached a mean size of $200 \mathrm{~mm}^{3}$. CT26.WT cells $\left(5 \times 10^{5}\right.$ cells/mouse $)$ in $0.1 \mathrm{ml}$ were injected subcutaneously into the right flank of mice (weight, $\sim 20 \mathrm{~g}$ ). When the tumors volume reached $\sim 100 \mathrm{~mm}^{3}$ on day 7 , the mice were treated orally ( $25 \mathrm{mg} / \mathrm{kg}$ LOR; $5 \mathrm{mg} / \mathrm{kg}$ THZ) every 2 days for 5 weeks. The sizes of the tumors were measured using calipers, and tumor volume $\left(\mathrm{mm}^{3}\right)$ was calculated according to the formula: Volume $=\left(\right.$ length $\mathrm{x}$ width $\left.{ }^{2}\right) / 2(11)$. All animal experiments were approved by the Laboratory Animal Welfare and Ethics Committee, School of Medicine, Shenzhen University (Shenzhen, China).

RNA extraction and quantitative reverse transcription polymerase chain reaction ( $R T-q P C R)$. Total RNA from CT26. WT cells and MFC cells was extracted using TRIzol (Thermo Fisher Scientific, Inc.) and was reverse transcribed using the Reverse Transcription system, High-Capacity cDNA Reverse Transcription kit, (Invitrogen; Thermo Fisher Scientific, Inc.) following the manufacturer's instructions. Reverse transcription was performed at $42^{\circ} \mathrm{C}$ for 60 min following incubation at $95^{\circ} \mathrm{C}$ for $5 \mathrm{~min}$. Amplification of specific products was performed with the following cycle profile: 1 cycle at $50^{\circ} \mathrm{C}$ for $2 \mathrm{~min}, 1$ cycle at $95^{\circ} \mathrm{C}$ for $10 \mathrm{~min}, 40$ cycles at $95^{\circ} \mathrm{C}$ for $15 \mathrm{sec}$ and $60^{\circ} \mathrm{C}$ for $1 \mathrm{~min}$, followed by the thermal denaturation protocol. RT-qPCR was performed using an MxPro Mx3000P Sequence Detection system (Stratagene; Agilent Technologies, Inc., Santa Clara, CA, USA) and Thunderbird SYBR qPCR mix (QPS-201; Toyobo Co., Ltd., Osaka, Japan). The primers were designed using Primer 5 software (PREMIER Biosoft International, Palo Alto, CA, USA). The primer sequences are as follows: Mouse PI3K forward, 5'-CACTCGTCACCATCA AACATGA-3' and reverse, 5'-AGGGTTGAAAAAGCCGAA GGT-3'; mouse Akt forward, 5'-ATGAACGACGTAGCCATT GTG-3' and reverse, 5'-TTGTAGCCAATAAAGGTGCCAT-3'; mouse mTOR forward, 5'-CAGTTCGCCAGTGGACTG AAG-3' and reverse, 5'-GCTGGTCATAGAAGCGAGTAG AC-3'. $\beta$-actin forward, 5'-GATCATTGCTCCTCCTGAGC-3' and reverse, 5'-ACTCCTGCTTGCTGATCCAC-3'. Transcript levels were calculated using the comparative $\mathrm{Cq}$ method $\left(2^{-\Delta \Delta \mathrm{Cq}}\right)$ and normalized to $\beta$-actin expression $(15,16)$.

Western blott analysis. Whole-cell lysates were harvested and lysed with M-PER Protein Extraction reagent (Pierce; Thermo Fisher Scientific, Inc.) with protease inhibitor cocktail (P8340, Sigma-Aldrich; Merck KGaA, Darmstadt, Germany). Equal quantities of the extracts $(10 \mu \mathrm{g})$ were resolved by $12 \%$ SDS/PAGE and electrophoretically transferred to polyvinylidene difluoride membranes (Bio-Rad Laboratories, Inc., Hercules, CA, USA). The membranes were blocked at room temperature for $1 \mathrm{~h}$ in TBS containing $0.05 \%$ Tween-20 (TBST) and 5\% non-fat milk powder, and then incubated overnight with primary antibodies $(1: 1,000)$ at $4^{\circ} \mathrm{C}$. Following three washes with TBST, the membranes were probed with the corresponding secondary antibody (Anti-rabbit IgG, horseradish peroxidase-linked antibody; cat. no. 7074; Cell Signaling Technology) for $1 \mathrm{~h}$ at room temperature. The membranes were washed again 3 times in TBST, and the protein were visualized using an Odyssey Infrared Imaging System (LI-COR Biosciences, Lincoln, NE, USA). 

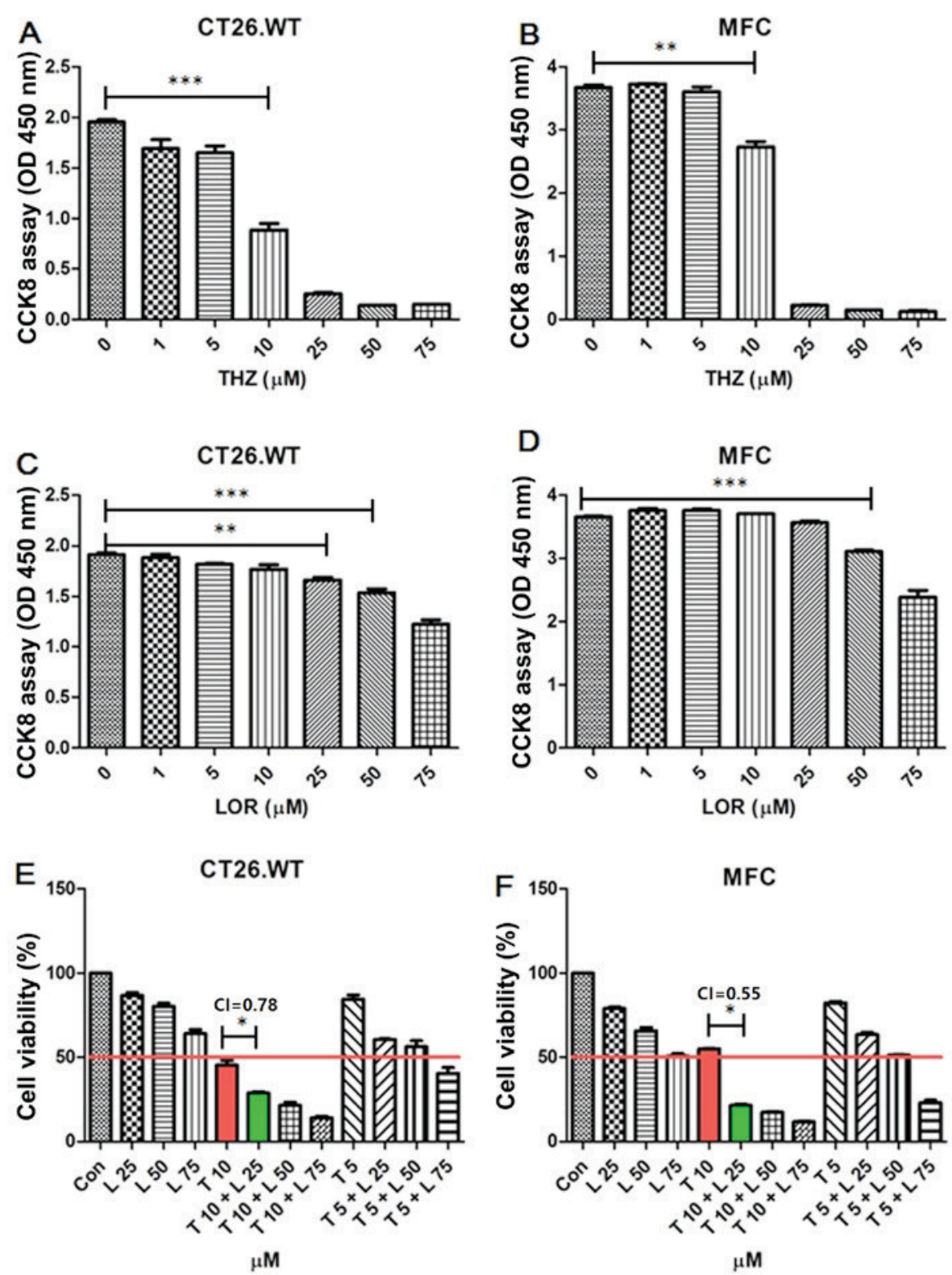

Figure 1. Thioridazine and loratadine inhibited proliferation of CT26.WT and MFC cells in vitro. (A and B) CT26.WT and MFC cells were treated with THZ $(0,1,5,10,25,50$ and $75 \mu \mathrm{M})$, and proliferation assay was performed at $24 \mathrm{~h}$. (C and D) CT26.WT and MFC cells were treated with LOR $(0,1,5,10,25,50$ and $75 \mu \mathrm{M}$ ), and proliferation assay was performed at $24 \mathrm{~h}$. Viability was measured by CCK-8 assay following treatment with various THZ concentrations plus LOR $(25,50$ and $75 \mu \mathrm{M})$ in (E) CT26.WT and (F) MFC cells. Combination index value was analyzed. CI $<1$ indicates a synergistic effect. All data represent the mean \pm standard deviation of $\geq 3$ independent experiments. ${ }^{*} \mathrm{P}<0.05,{ }^{* * *} \mathrm{P}<0.01,{ }^{* * *} \mathrm{P}<0.001$. Con, control; LOR, loratadine; THZ, thioridazine; OD, optical density.

Statistical analysis. Data are presented as the mean \pm standard deviation. Student's t-test was used for comparisons of two independent groups. For comparisons of $\geq 3$ groups, one-way analysis of variance was performed. GraphPad-Prism 5.0 (GraphPad Software, Inc., La Jolla, CA, USA) was used to perform the statistical analyses. $\mathrm{P}<0.05$ was considered to indicate a statistically significant difference.

\section{Results}

Treatment with THZ and LOR inhibits the growth of mouse gastrointestinal carcinoma cells in vitro. To investigate the cytotoxic effect of THZ and LOR on mouse gastrointestinal carcinoma cells, CT26.WT and MFC cells were treated with various concentrations of THZ or LOR for $24 \mathrm{~h}$, viability was then measured by using CCK- 8 assay. Both drugs were demonstrated to have an inhibitory effect on the growth of the cells in a dose-dependent manner (Fig. 1). Furthermore, treatment with $10 \mu \mathrm{M}$ THZ or $50 \mu \mathrm{M}$ LOR resulted in approximately 30-40\% reduction in viability of CT26.WT cancer cells (Fig. 1A and C) as well as MFC cells (Fig. 1B and D).

To further determine the potential synergistic growth-inhibitory effects of THZ and LOR combination treatment, the viability of the cells treated with single and combined treatment were analyzed using the $\mathrm{CI}$ value (Fig. 1E and F). Treatment with a combination of THZ $(10 \mu \mathrm{M})$ and LOR $(25 \mu \mathrm{M})$ resulted in synergistic inhibition, where the CI value was 0.78 for CT26.WT cells and 0.55 for MFC cells. 


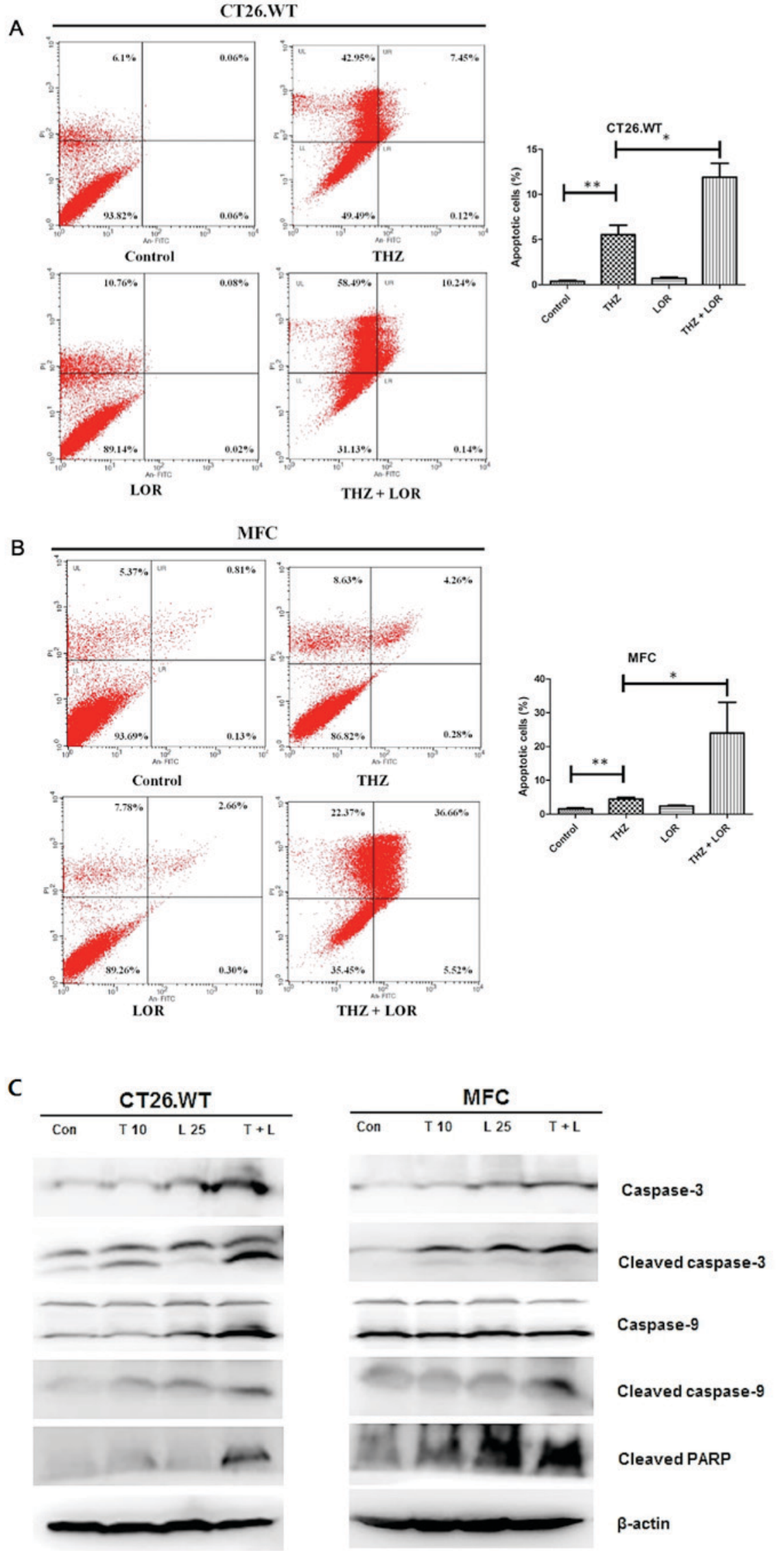

Figure 2. CT26.WT cells were treated with the combination of $10 \mu \mathrm{M} \mathrm{THZ}$ and $25 \mu \mathrm{M} \mathrm{LOR}$, and apoptosis assay was performed at $24 \mathrm{~h}$. (A) CT26.WT cells were incubated with THZ and/or LOR treatment for $24 \mathrm{~h}$, followed by annexin V/propidium iodide double staining to evaluate early- and late-stage apoptosis (B) Similar procedures were performed in MFC cells. The histograms indicate the percentage of apoptotic cells, and data represent the mean \pm standard deviation of $\geq 3$ independent experiments. (C) The level of caspase-3, -9 and cleaved-PARP expression was detected by western blotting. $\beta$-actin was used as a loading control. ${ }^{*} \mathrm{P}<0.05,{ }^{* *} \mathrm{P}<0.01$. LOR, loratadine; THZ, thioridazine; PARP, poly (ADP-ribose) polymerase 1. 

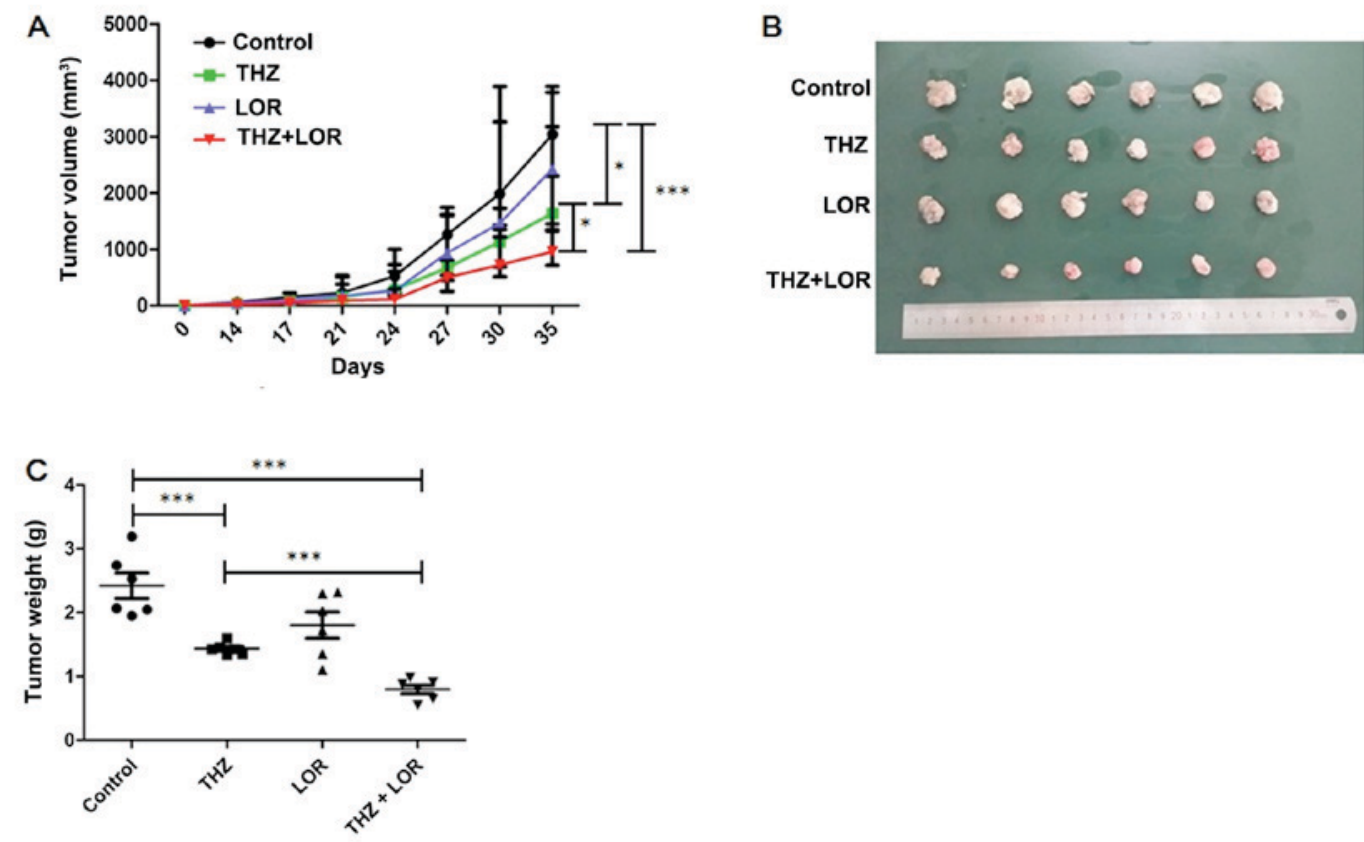

Figure 3. Combination treatment with THZ and LOR inhibited tumor growth in vivo. (A) Growth curves of tumors derived from CT26.WT cells, which were pretreated with THZ and/or LOR. (B) Morphology of tumors treated with THZ, LOR or a combination of THZ and LOR on day 35. (C) The weight of excised tumors from each treatment group was measured on day $35 .{ }^{*} \mathrm{P}<0.05,{ }^{* * * *} \mathrm{P}<0.001$. Con, control; LOR, loratadine; THZ, thioridazine.

$10 \mu \mathrm{M}$ THZ and $25 \mu \mathrm{M}$ LOR were selected for further studies investigating the mechanism of action.

Treatment with THZ and LOR induces apoptosis. To detect whether treatment with THZ or LOR or THZ and LOR combination treatment induced gastrointestinal cancer cell apoptosis, annexin V/PI double staining assay was performed. The data revealed that the combination treatment resulted in an increase in the percentage of early (lower right) and late (upper right) apoptosis compared with the control. The total percentage of apoptosis (sum of early and late stage) was $11.92 \pm 2.7 \%$ in the combination-treated group, compared with $5.5 \pm 1.8 \%$ in the THZ treatment group of CT26.WT cells (Fig. 2A, P<0.05). The effects of THZ and LOR treatment in MFC cells were also compared, and similar apoptosis patterns between the two treatment groups were observed (Fig. $2 \mathrm{~B}, \mathrm{P}<0.05$ ).

Subsequently, it was analyzed whether this effect on apoptosis was associated with the activation of caspase-3. Western blotting revealed that treatment with THZ and LOR markedly increased caspase-3, -9 and PARP cleavage in CT26.WT and MFC cells (Fig. 2C), suggesting that the combination treatment induced apoptosis of gastrointestinal cancer cells in a caspase-dependent manner.

Treatment with THZ and LOR inhibits mouse colon cancer cell proliferation in vivo. Next, it was investigated whether the combination of $\mathrm{THZ}(5 \mathrm{mg} / \mathrm{kg})$ (7) and loratadine $(25 \mathrm{mg} / \mathrm{kg})(10) \mathrm{had}$ higher rates of therapeutic efficacy compared with $T H Z$ or LOR treatments for BALB/c mice bearing CT26.WT tumor xenografts. Although treatment with $\mathrm{THZ}$ alone resulted in a reduction in tumor growth, the combination treatment was more effective in inhibiting tumor growth compared with treatment alone with THZ or LOR (Fig. 3A). In addition, the combination treatment significantly reduced tumor weight and reduced volume $>50 \%$ compared with the control mice (Fig. 3A-C).

Synergistic suppression of the PI3K/Akt/mTOR signaling pathway by combination treatment with THZ and LOR. THZ has been previously shown to induce apoptosis by suppressing the PI3K/Akt/mTOR signaling pathway in cervical and endometrial cancer cells (12). Therefore, it was investigated whether the effects of the combination treatment may also be mediated via the PI3K/Akt/mTOR signaling pathway.

The level of PI3K, Akt and mTOR mRNA was quantified by qRT-PCR (Fig. 4). Treatment with THZ alone resulted in decreased transcription of PI3K (Fig. 4A and B), Akt (Fig. 4C and D) and mTOR (Fig. 4E and F) in CT26.WT and MFC cells compared with control $(\mathrm{P}<0.05)$, but the effects of the combination treatment was more significant compared with THZ alone $(\mathrm{P}<0.05)$.

The phosphorylation of Akt at Ser473, PI3K at Tyr458 and mTOR at Ser2448 was also verified using western blotting (Fig. 4G). As expected, following combination treatment, the cells exhibited a marked decrease in phosphorylation of PI3K, Akt and mTOR. Taken together, these data suggest that combination treatment targets the PI3K/Akt/mTOR signaling pathway, which leads to the inhibition of tumor growth.

\section{Discussion}

Due to the limited efficiency of single agents in cancer treatment, there is growing evidence indicating that combination therapies can augment the effectiveness of single agents (4). 

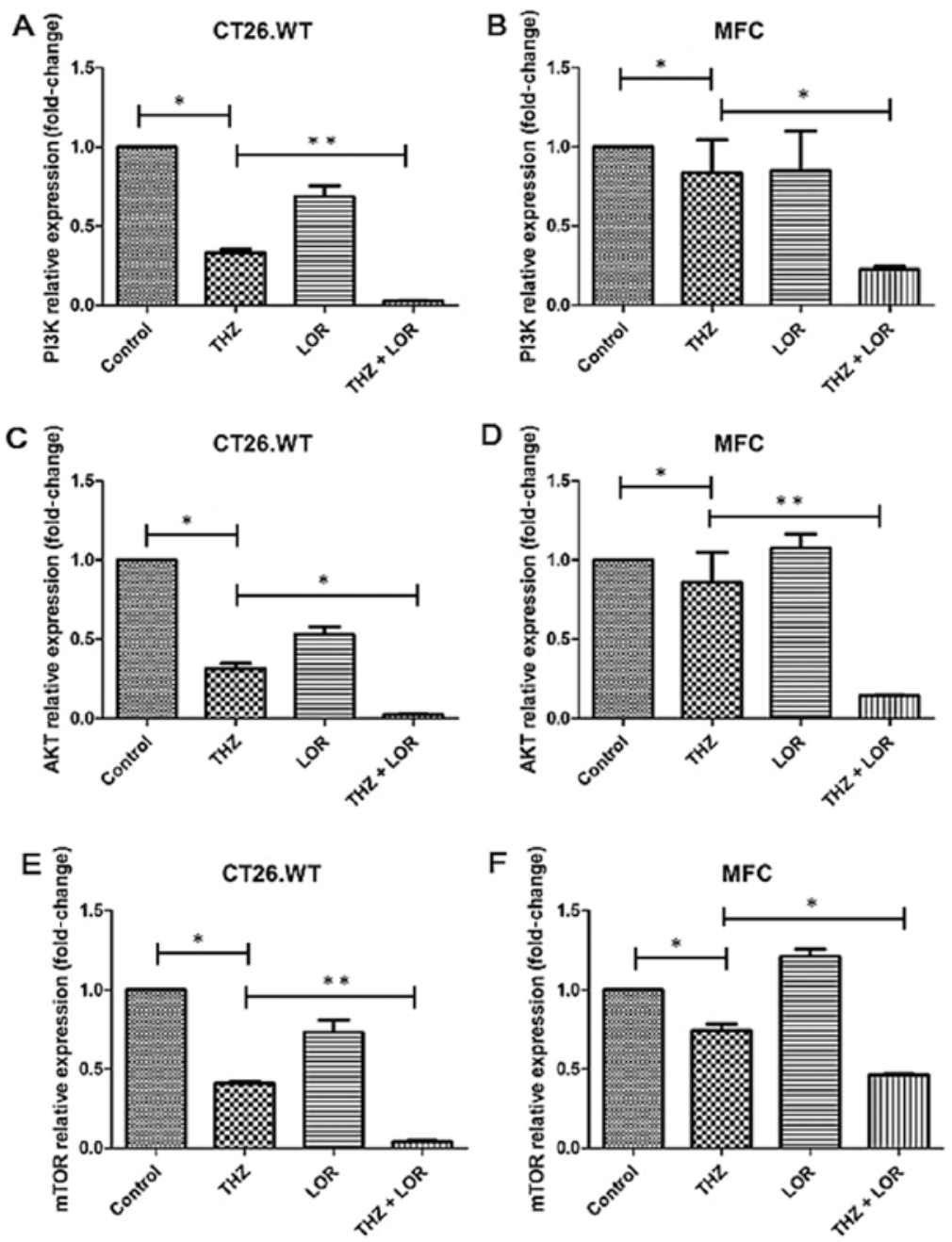

G
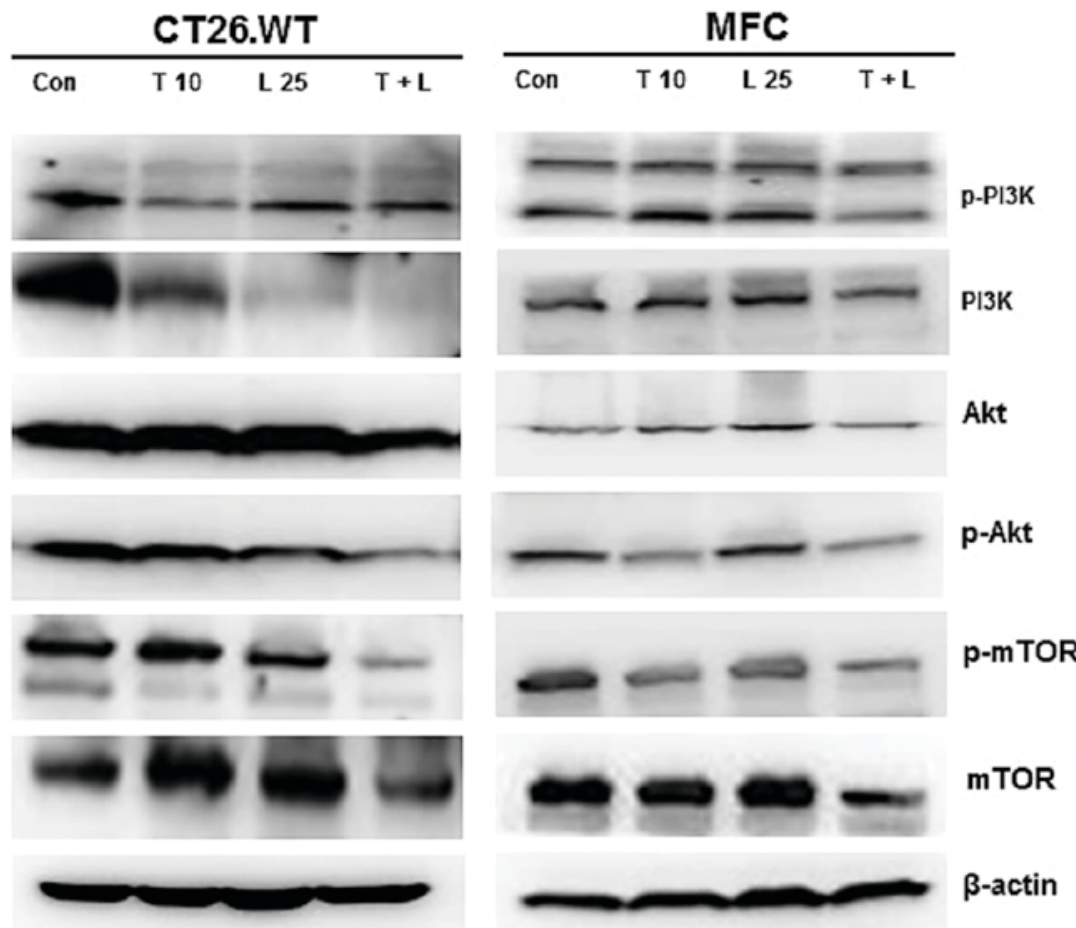

Figure 4. Western blot analysis of PI3K/Akt/mTOR signaling pathways. Quantitative reverse transcription polymerase chain reaction demonstrated that decreased expression of (A and B) PI3K, (C and D) Akt and (E and F) mTOR in CT26.WT and MFC cells in the combination treatment group compared with the THZ treatment group. (G) Combination therapy decreased the phosphorylation of major components of the PI3K/Akt/mTOR signaling pathways. Total cell lysates were prepared and analyzed by immunoblotting using antibodies against p-PI3K, p-Akt and p-mTOR. ${ }^{*} \mathrm{P}<0.05,{ }^{* *} \mathrm{P}<0.01$. Con, control; LOR, loratadine; mTOR; mammalian target of rapamycin; p, phosphorylated; PI3K, phosphoinositide 3-kinase; THZ, thioridazine. 
THZ, which is used extensively for the treatment of psychotic diseases, has been reported to selectively target leukemic cancer stem cells (8) and have demonstrated angiostatic effects mediated via inhibition of the vascular endothelial growth factor receptor-2/PI3K/mTOR signaling pathway in ovarian cancer xenografts (11). THZ is also capable of inducing cancer cell apoptosis in various types of cancer (17-19). LOR, an oral anti-histamine agent used worldwide, has been demonstrated to mediate antitumor effects by arresting human colon cancer COLO 205 cells at the G2/M phase (13).

In the present study, it was demonstrated that treatment with a combination of THZ and LOR dramatically suppressed growth in colon and gastric cancer cells by inducing apoptosis. The mechanisms were mediated by inhibition of the $\mathrm{PI} 3 \mathrm{~K} / \mathrm{Akt} / \mathrm{mTOR}$ signaling pathway. Furthermore, in BALB/c mice bearing CT26.WT colon tumor xenografts, the combination treatment inhibited tumor growth more effectively compared with treatment with THZ alone and LOR alone, respectively. As expected, the volume of tumors treated with THZ and LOR was $70 \%$ smaller compared with the volume of the controls. These results indicate that the combination treatment with $\mathrm{THZ}$ and LOR was able to mediate anti-gastrointestinal cancer effects.

When evaluating the effect of the agents on cell growth, it was observed that only relatively high concentrations of THZ $(10 \mu \mathrm{M})$ or LOR $(50 \mu \mathrm{M})$ alone exhibited cytotoxicity against CT26.WT and MFC cells. However, treatment with a combination of THZ $(10 \mu \mathrm{M})$ and LOR $(25 \mu \mathrm{M})$ resulted in a synergistic effect, with a CI value of 0.78 for CT26.WT cells and 0.55 for MFC cells.

Based on these findings, the effect of the combination treatment on apoptosis was further investigated. It was observed that the combination treatment resulted in a marked increase in the percentage of apoptotic cells compared with treatment with single drugs.

It has been previously demonstrated that induction of tumor cell apoptosis is the most effective therapeutic strategy for eliminating cancer cells $(20,21)$. The mechanisms of the apoptosis response involve caspase activation, specifically the expression of caspase-3 and 9, which are the key components of apoptosis response (22). In the present study, following combination treatment with THZ and LOR, the expression of cleaved forms of caspase-3 and 9 was markedly higher compared with the control, indicating greater caspase-3 and caspase- 9 activities and increased apoptotic capability. The expression of cleaved PARP, which is another downstream effector, was also upregulated when treated with a combination of THZ and LOR compared with the control.

A previous study revealed that $\mathrm{PI} 3 \mathrm{~K} / \mathrm{Akt} / \mathrm{mTOR}$ signaling pathway is inhibited by THZ in cervical and endometrial cells (11). PI3K/Akt/mTOR is one of the major signaling pathways that have been identified to exert key roles in various physiological processes in human malignant tumors (23). Upregulation of the PI3K/Akt/mTOR signaling pathway has been demonstrated to reduce apoptosis of tumor cells and increase proliferation. Therefore, inhibition of the pathway suppresses tumor cell growth and increases the cytotoxic effect of conventional chemotherapeutic agents in numerous solid tumors $(24,25)$. PI3K coordinates cell growth, cell cycle entry, cell migration and cell survival (26). Akt activity is modulated by phosphorylation via PI3K, which recruits Akt to the plasma membrane (27). Activation of Akt results in cell cycle progression, survival, migration and metabolism through phosphorylation of a number of downstream effectors, including caspase-9 (28), forkhead transcription factors (29) and mTOR (30). mTOR is a Ser/Thr protein kinase $(31,32)$ that functions as an ATP and amino acid sensor to regulate tumor growth, cell proliferation and protein synthesis $(33,34)$. In the present study, RT-qPCR results indicated that the expression of PI3K, Akt and mTOR mRNA was significantly decreased when the cells were treated with THZ and LOR together compared with cells treated with THZ alone. Additionally, the protein levels of activated phosphorylated forms of PI3K, Akt and mTOR were also downregulated when treated with combination therapy compared with cells treated with THZ alone.

In summary, to the best of our knowledge, the present study is the first to report that treatment with a combination of THZ and LOR exhibited favorable anti-gastrointestinal cancer effects in in vitro and in vivo experiments, suggesting that it may be a potential strategy for the treatment of gastrointestinal tumors.

\section{Acknowledgements}

The present study was supported by grants from the Shenzhen Nanshan District Biotech Key Project (grant no. KC2013JSJS0017A), the Shenzhen City Biotech Key Project (grant no. JSGG20120614151715670) and the China Postdoctoral Science Foundation (grant no. 2015M572368).

\section{References}

1. Chen W, Zheng R, Zhang S, Zeng H, Xia C, Zuo T, Yang Z, Zou X and He J: Cancer incidence and mortality in China, 2013. Cancer Lett 401: 63-71, 2017.

2. Parkin DM, Bray F, Ferlay J and Pisani P: Global cancer statistics, 2002. CA Cancer J Clin 55: 74-108, 2005.

3. Fitzgerald JB, Schoeberl B, Nielsen UB and Sorger PK: Systems biology and combination therapy in the quest for clinical efficacy. Nat Chem Biol 2: 458-466, 2006.

4. Schnipper LE, Davidson NE, Wollins DS, Tyne C, Blayney DW, Blum D, Dicker AP, Ganz PA, Hoverman JR, Langdon R, et al: American society of clinical oncology statement: A conceptual framework to assess the value of cancer treatment options. J Clin Oncol 33: 2563-2577, 2015.

5. Sgambato D, Capuano A, Sullo MG, Miranda A, Federico A and Romano M: Gut-brain axis in gastric mucosal damage and protection. Curr Neuropharmacol 14: 959-966, 2016.

6. Grubišić V, Verkhratsky A, Zorec R and Parpura V: Enteric glia regulate gut motility in health and disease. Brain Res Bull: Mar 29, 2017 (Epub ahead of print). doi: 10.1016/j.brainresbull.2017.03.011.

7. Keightley PC, Koloski NA and Talley NJ: Pathways in gut-brain communication: Evidence for distinct gut-to-brain and brain-to-gut syndromes. Aust N Z J Psychiatry 49: 207-214, 2015.

8. Sachlos E, Risueño RM, Laronde S, Shapovalova Z, Lee JH, Russell J, Malig M, McNicol JD, Fiebig-Comyn A, Graham M, et al: Identification of drugs including a dopamine antagonist that selectively target cancer stem cells. Cell 149: 1284-1297, 2012.

9. Mu J, Xu H, Yang Y, Huang W, Xiao J, Li M, Tan Z, Ding Q, Zhang L, Lu J, et al: Thioridazine, an antipsychotic drug, elicits potent antitumor effects in gastric cancer. Oncol Rep 31: 2107-2114, 2014.

10. Ke XY, Lin Ng VW, Gao SJ, Tong YW, Hedrick JL and Yang YY: Co-delivery of thioridazine and doxorubicin using polymeric micelles for targeting both cancer cells and cancer stem cells. Biomaterials 35: 1096-1108, 2014.

11. Park MS, Dong SM, Kim BR, Seo SH, Kang S, Lee EJ, Lee SH and Rho SB: Thioridazine inhibits angiogenesis and tumor growth by targeting the VEGFR-2/PI3K/mTOR pathway in ovarian cancer xenografts. Oncotarget 5: 4929-4934, 2014. 
12. Kang S, Dong SM, Kim BR, Park MS, Trink B, Byun HJ and Rho SB: Thioridazine induces apoptosis by targeting the $\mathrm{PI} 3 \mathrm{~K} / \mathrm{Akt} / \mathrm{mTOR}$ pathway in cervical and endometrial cancer cells. Apoptosis 17: 989-997, 2012.

13. Chen JS, Lin SY, Tso WL, Yeh GC, Lee WS, Tseng H, Chen LC and Ho YS: Checkpoint kinase 1-mediated phosphorylation of $\mathrm{Cdc} 25 \mathrm{C}$ and bad proteins are involved in antitumor effects of loratadine-induced $\mathrm{G} 2 / \mathrm{M}$ phase cell-cycle arrest and apoptosis. Mol Carcinog 45: 461-478, 2006.

14. Chou TC: Drug combination studies and their synergy quantification using the Chou-Talalay method. Cancer Res 70: 440-446, 2010.

15. Lin Y, Zhang H, Liang J, Li K, Zhu W, Fu L, Wang F, Zheng X, Shi $\mathrm{H}, \mathrm{Wu} \mathrm{S}$, et al: Identification and characterization of alphavirus $\mathrm{M} 1$ as a selective oncolytic virus targeting ZAP-defective human cancers. Proc Natl Acad Sci USA 111: E4504-E4512, 2014.

16. Livak KJ and Schmittgen TD: Analysis of relative gene expression data using real time quantitative PCR and the 2(-Delta Delta C(T)) method. Methods 25: 402-408, 2001.

17. Strobl JS, Kirkwood KL, Lantz TK, Lewine MA, Peterson VA and III: Inhibition of human breast cancer cell proliferation in tissue culture by the neuroleptic agents pimozide and thioridazine. Cancer Res 50: 5399-5405, 1990.

18. Gil-Ad I, Shtaif B, Levkovitz Y, Dayag M, Zeldich E and Weizman A: Characterization of phenothiazine-induced apoptosis in neuroblastoma and glioma cell lines: Clinical relevance and possible application for brain-derived tumors. J Mol Neurosci 22: 189-198, 2004.

19. Zhelev Z, Ohba H, Bakalova R, Hadjimitova V, Ishikawa M, Shinohara Y and Baba Y: Phenothiazines suppress proliferation and induce apoptosis in cultured leukemic cell without any influence on the viability of normal lymphocytes. Phenothiazines and leukemia. Cancer Chemother Pharmacol 53: 267-275, 2004.

20. Plati J, Bucur O and Khosravi-Far R: Apoptotic cell signaling in cancer progression and therapy. Integr Biol (Camb) 3: 279-296, 2011.

21. Wong RS: Apoptosis in cancer: From pathogenesis to treatment. J Exp Clin Cancer Res 30: 87, 2011.
22. Riedl SJ and Shi Y: Molecular mechanisms of caspase regulation during apoptosis. Nat Rev Mol Cell Biol 5: 897-907, 2004.

23. de Melo AC, Paulino E and Garces ÁH: A review of mTOR pathway inhibitors in gynecologic cancer. Oxid Med Cell Longev 2017: 4809751, 2017.

24. Hu L, Hofmann J and Jaffe RB: Phosphatidylinositol 3-kinase mediates angiogenesis and vascular permeability associated with ovarian carcinoma. Clin Cancer Res 11: 8208-8212, 2005.

25. Shi Y, Frankel A, Radvanyi LG, Penn LZ, Miller RG and Mills GB: Rapamycin enhances apoptosis and increases sensitivity to cisplatin in vitro. Cancer Res 55: 1982-1988, 1995.

26. Cantley LC: The phosphoinositide 3-kinase pathway. Science 296 : 1655-1657, 2002

27. King D, Yeomanson D and Bryant HE: PI3King the lock: Targeting the PI3K/Akt/mTOR pathway as a novel therapeutic strategy in neuroblastoma. J Pediatr Hematol Oncol 37: 245-251, 2015.

28. Cardone MH, Roy N, Stennicke HR, Salvesen GS, Franke TF, Stanbridge E, Frisch S and Reed JC: Regulation of cell death protease caspase-9 by phosphorylation. Science 282: 1318-1321, 1998.

29. Brunet A, Bonni A, Zigmond MJ, Lin MZ, Juo P, Hu LS, Anderson MJ, Arden KC, Blenis J and Greenberg ME: Akt promotes cell survival by phosphorylating and inhibiting a Forkhead transcription factor. Cell 96: 857-868, 1999.

30. Rafalski VA and Brunet A: Energy metabolism in adult neural stem cell fate. Prog Neurobiol 93: 182-203, 2011.

31. Brown EJ, Albers MW, Shin TB, Ichikawa K, Keith CT, Lane WS and Schreiber SL: A mammalian protein targeted by G1-arresting rapamycin-receptor complex. Nature 369: 756-758, 1994.

32. Sabatini DM, Erdjument-Bromage H, Lui M, Tempst P and Snyder SH: RAFT1: A mammalian protein that binds to FKBP12 in a rapamycin-dependent fashion and is homologous to yeast TORs. Cell 78: 35-43, 1994.

33. Wan X, Harkavy B, Shen N, Grohar P and Helman LJ: Rapamycin induces feedback activation of Akt signaling through an IGF-1R-dependent mechanism. Oncogene 26: 1932-1940, 2007.

34. Dennis PB, Jaeschke A, Saitoh M, Fowler B, Kozma SC and Thomas G: Mammalian TOR: A homeostatic ATP sensor. Science 294: 1102-1105, 2001. 